\title{
ACESSIBILIDADE AO SERVIÇO DE SAÚDE DA MULHER NO CICLO GRAVÍDICO- PUERPERAL
}

\author{
Sheila Milena Pessoa dos Santos ${ }^{1}$, Lais Vasconcelos Santos ${ }^{2}$, Jéssica Oliveira Rodrigues ${ }^{3}$, Lara Caline Santos \\ Lira $^{2}$, Laianne Barbosa de Souza², Ueigla Batista da Silva²
}

RESUMO: Objetivou-se analisar a acessibilidade ao serviço de saúde da mulher no ciclo gravídico-puerperal. Tratase de um estudo documental, exploratório e descritivo, com abordagem quantitativa. A população foi composta por 69 mulheres que estiveram em período gravídico ou puerperal no período de janeiro de 2012 a abril de 2014. $\mathrm{Na}$ coleta de dados utilizou-se um instrumento semiestruturado. Para a análise, adotou-se estatística descritiva e a literatura pertinente à temática. Diante os achados, identificou-se deficiência nos registros de prontuários e cartões das gestantes; ausência de consulta de pré-natal com médicos; baixos percentuais dos procedimentos de inspeção de mamas, exame ginecológico e consulta odontológica; baixa realização dos exames preconizados no programa Rede Cegonha, vinculado ao município. Pretende-se que esta pesquisa sirva de subsídio para compreensão do funcionamento das ações atuais, reconhecimento das barreiras de acessibilidade existentes e possibilidade da criação de estratégias para melhorar a assistência prestada durante o ciclo gravídico-puerperal. DESCRITORES: Saúde da mulher; Acessibilidade aos serviços de saúde; Assistência pré-natal.

\section{ACCESSIBILITY TO WOMEN'S HEALTHCARE SERVICE DURING PREGNANCY AND POSTPARTUM}

ABSTRACT: The objective of this study was to analyze accessibility to women's healthcare service during pregnancy and postpartum. A documental, exploratory and descriptive study, with a quantitative approach, was conducted with a sample of 69 women who were pregnant or in postpartum, from January 2012 to April 2014. A semi-structured questionnaire was used for data collection, and descriptive statistics and specific literature were used for data analysis. We identified poor medical records; women with no prenatal consultations; a low number of women who were submitted to breast inspection procedures, gynecological examination and dentist appointments; and a low number of accomplished tests recommended by the Rede Cegonha program, linked to the municipality. This research is expected to serve as support to understand how current medical actions work and to acknowledge the existence of accessibility barriers to woman's healthcare service and the possibility of creating new strategies to improve medical care during pregnancy and postpartum.

DESCRIPTORS: Women's health; Health services accessibility; Prenatal care.

\section{ACCESIBILIDAD AL SERVICIO DE SALUD DE LAMUJER EN EL CICLO EMBARAZO-PUERPERIO}

RESUMEN: Se objetivó analizar la accesibilidad al servicio de salud de la mujer en el ciclo embarazo-puerperio. Estudio documental exploratorio y descriptivo, con abordaje cuantitativo. Población compuesta por 69 mujeres que estuvieron en período de embarazo o puerperal entre enero de 2012 y abril de 2014. Datos recolectados mediante instrumento semiestructurado; analizados por estadística descriptiva y literatura pertinente a la temática. Entre los hallazgos, se identificó deficiencia en registro de historias clínicas y fichas de embarazadas; ausencia de consulta prenatal con médicos; bajos porcentajes de procedimientos de inspección mamaria, examen ginecológico y consulta odontológica; baja realización de los análisis recomendados en el programa Rede Cegonha, impulsado por el municipio. Se pretende que la investigación colabore en la comprensión del funcionamiento de las acciones actuales, el reconocimiento de los obstáculos de accesibilidad existentes y la posibilidad de creación de estrategias que mejoren la atención brindada durante el ciclo embarazo-puerperio.

DESCRIPTORES: Salud de la Mujer; Accesibilidad a los Servicios de Salud; Atención Prenatal.

${ }^{1}$ Enfermeira. Doutoranda em Enfermagem. Docente de Enfermagem da Universidade Federal de Campina Grande. Campina Grande, PB, Brasil.

${ }^{2}$ Discente de Enfermagem. Universidade Federal de Campina Grande. Campina Grande, PB, Brasil.

${ }^{3}$ Enfermeira. Universidade Federal de Campina Grande. Campina Grande, PB, Brasil. 


\section{- INTRODUÇÃO}

A Organização Mundial da Saúde estima que, em 2010, cerca de 287 mil mulheres no mundo morreram durante a gestação e o parto. Dessas mortes, a maioria se concentra nos países em desenvolvimento e são decorrentes da falta de acesso a cuidados de rotina adequados e cuidados de emergência quando necessários. Percebe-se um declínio quando comparado a dados de 1990, entretanto, tais valores estão distantes da $5^{\circ}$ meta dos Objetivos de Desenvolvimento do Milênio de redução de $75 \%$ das mortes maternas até $2015^{(1)}$.

Nesta direção, para melhorar a assistência à gestante e recém-nascido, deve-se planejar ações considerando a identificação de fatores de risco relacionados com a mortalidade materna e neonatal, pois tal situação é um importante indicador que reflete as condições socioeconômicas, reprodutivas e, principalmente, as relacionadas à assistência pré-natal, ao parto e aos recém-nascidos ${ }^{(2)}$.

A assistência pré-natal, quando ofertada com qualidade, é compreendida como disponibilidade de infraestrutura adequada no que se refere aos recursos físicos, materiais, humanos e financeiros, atendimento multidisciplinar, orientações e condutas que atendam às necessidades de cada gestante (3). Assim, entende-se que a atenção às mulheres grávidas deve ser pautada não só em procedimentos clínicos, mas em um conjunto de ações como promoção da saúde, acolhimento, estabelecimento de vínculo, entre outras tecnologias de forma a desenvolver a autonomia da mulher para o seu autocuidado $^{(4)}$.

Embora o acesso ao pré-natal alcance praticamente toda a população, a qualidade desse atendimento ainda é insatisfatória visto que atividades educativas em saúde não abordam as necessidades reprodutivas e sexuais de mulheres e homens, a rede de serviços de apoio diagnóstico para realização de exames preconizados durante a gravidez não contempla as necessidades dos municípios, além de existir em diversas localidades a peregrinação da mulher para encontrar um estabelecimento de saúde no momento de atenção ao parto e nascimento ${ }^{(5)}$.

Diante de tais conformações, a avaliação da qualidade do serviço serve como norteadora para equipe e gestores para mensurar a efetividade do serviço. Assim, a análise da acessibilidade constitui importante dimensão para estudos que investigam o desempenho dos serviços de saúde e a qualidade da atenção prestada. Essas análises são fundamentais, visto que a consideração dos resultados encontrados à luz dos avanços teóricos relativos à organização setorial podem transformar práticas no interior dos serviços ${ }^{(6-7)}$.

No contexto atual, a problemática da acessibilidade insuficiente à assistência gestacional acarreta vulnerabilidade materno-infantil. Ao mesmo tempo, torna tanto o período puerperal quanto o gestacional desarticulados e fragmentados. Sendo assim, deve-se investir na investigação dessas lacunas para propor estratégias de superação dos entraves identificados.

Face ao exposto, pretende-se analisar a acessibilidade ao serviço de saúde da mulher no ciclo gravídico puerperal.

\section{METODOLOGIA}

Trata-se de um estudo documental, exploratório e descritivo, com abordagem quantitativa, elaborado a partir do projeto intitulado "Acessibilidade ao serviço no ciclo gravídico-puerperal: Uma avaliação da atenção à mulher na atenção básica", vinculado ao Programa Nacional de Reorientação da Formação Profissional em Saúde articulado ao Programa de Educação pelo Trabalho para a Saúde da linha de ação Rede Cegonha, realizado no município de Lagoa Seca, Paraíba, em parceria com a Universidade Federal de Campina Grande, vigência 2012-2014.

O estudo foi realizado em duas Unidades de Saúde da Família (USF), do município de Lagoa Seca, Paraíba. A população foi composta por mulheres que estiveram/estavam em período gravídico ou puerperal no período de janeiro de 2012 a abril de 2014 e que realizaram pré-natal nas unidades participantes do estudo, não havendo limitação de idade, cor/raça ou deficiência física permanente 
ou transitória. O universo considerou um levantamento de gestantes a partir dos registros de cada serviço, no recorte temporal adotado, resultando em: USF A 125 gestantes, USF B 102 gestantes.

A amostra foi calculada tomando como base a fórmula de determinação do tamanho mínimo de amostra para proporção de uma população finita: $n=\frac{N \cdot p \cdot q \cdot\left(Z \alpha_{2}\right)^{2}}{p \cdot q \cdot\left(Z \alpha_{2}\right)^{2}+(N-1) E^{2}}$

na qual $\mathrm{n}=$ Número de indivíduos na amostra, $\mathrm{N}=$ Tamanho da população, $\mathrm{p}=$ Proporção populacional estudada, $\mathrm{q}=$ Proporção populacional não estudada, $\mathrm{Za} / 2=$ Grau de confiança desejado, $\mathrm{E}=$ Margem de erro. Adotou-se nível de confiança de $98 \%$ e margem de erro de $2 \%$. O qual proporcionou grupos de 38 e 31 mulheres para USF A e USF B, respectivamente.

A fonte de documentos primários deste estudo foi composta por prontuários e/ou fichas e os cartões de gestante das mulheres pesquisadas. Realizou-se um sorteio para organizar a ordem de inclusão e busca das participantes. Como critério de exclusão, adotou-se a residência incompatível com a área de abrangência das USF. As 69 mulheres selecionadas concordaram em participar da pesquisa e disponibilizaram seus prontuários e cartões das gestantes para fonte deste estudo. Quanto aos cartões, nove delas não possuíam e relataram haver perdido.

O instrumento de coleta foi adaptado de um formulário ${ }^{(8)}$, considerando a ficha perinatal ${ }^{(9)}$, cartão da gestante ${ }^{(10)}$ e indicadores estratégicos para a Rede Cegonha. O mesmo possui questões de identificação, história obstétrica e avaliação de acessibilidade para assistência pré-natal.

Os dados advindos da coleta dos documentos alimentaram um banco de dados divididos por fonte realizados no programa estatístico EpilnfoTM versão7. Após a digitação, foram revisados e tabulados. Para a análise, adotou-se a estatística descritiva, compondo variáveis para perfil sociodemográfico (faixa etária, escolaridade, estado civil, raça, ocupação); perfil obstétrico [quantidade de gestações, $\mathrm{n}^{\circ}$ de filhos, tipo de parto (anterior e atual)]; avaliação da acessibilidade ( $\mathrm{N}^{\circ}$ de consultas, procedimentos do atendimento, exames realizados, assistência puerperal). Os resultados foram discutidos à luz da literatura que versa sobre acessibilidade, assistência pré-natal, parto e puerpério.

O projeto foi encaminhado ao Comitê de Ética e Pesquisado Hospital Universitário Alcides Carneiro e aprovado sob parecer $\mathrm{n}^{\circ}$ 869.561. Somente após a aprovação pelo comitê foi iniciada a coleta de dados da pesquisa.

\section{RESULTADOS}

Para composição sociodemográfica, abordaram-se as variáveis relativas à idade, escolaridade, estado civil, raça e ocupação. Salienta-se que o preenchimento incompleto do cartão da gestante e/ou dados não anotados no prontuário dificultou a coleta de determinadas variáveis, bem como a análise.

Participaram do estudo 69 mulheres, tendo predomínio da faixa etária de 21-25 anos (31,67\% cartão e $26,09 \%$ prontuário), casadas convivendo com companheiros e filhos (65\% cartão e 53,62\% prontuário) e com ensino médio ( $50 \%$ cartão e $39,13 \%$ prontuário). Em relação à variável raça, deparou-se neste estudo com uma divergência de preenchimento nas fontes, alcançando a ausência dessa informação em $13,33 \%$ o cartão das gestantes. Nos prontuários, o não preenchimento alcançou 89,86\%. Para o cartão, houve maior número de mulheres brancas (36,67\%) e pretas $(26,67 \%)$.

Quanto à ocupação, variável encontrada no prontuário, percebe-se predomínio das agricultoras $\mathrm{n}=35(50,72 \%)$, possivelmente relacionado à localização de moradia que consiste em zona rural $\mathrm{n}=51$ $(73,91 \%)$.

Referente ao perfil obstétrico, verificou-se que para número de gestações, as mulheres eram na maioria primigestas (30\% cartão e $36,23 \%$ prontuário) e secundigestas $(31,67 \%$ cartão e $28,99 \%$ prontuário). Percebeu-se variação de um a quatro filhos, sobressaindo as que possuíam um filho (26,67\% cartão e $24,64 \%$ prontuário). Quanto ao tipo de parto anterior, houve predomínio dos partos vaginais (46,67\% cartão e 40,58\% prontuário).

Quanto ao preenchimento do tipo de parto, verificou-se o não preenchimento das informações dos 
cartões das gestantes nas maternidades $(76,74 \%)$.

Houve ocorrência de óbitos na primeira semana (2,90\%) e a ausência de registros de natimortos e óbitos após primeira semana. Referente a abortos, houve registro $(7,25 \%)$ no cartão da gestante, sendo 2,90\% espontâneos.

Para a caracterização do atendimento pré-natal, adotou-se as seguintes variáveis: número de consultas, número de consultas por profissional, início do pré-natal, procedimentos de rotina (aferição de pressão arterial (PA), dados antropométricos, vacinação, inspeção de mamas, realização de exame ginecológico, participação consulta odontológica), exames realizados (primeira solicitação e repetições) e consulta puerperal.

Referente à quantidade de consultas realizadas durante o pré-natal, notou-se predomínio de mais de seis consultas (58,33\% cartão e 57,97\% prontuário), iniciadas no primeiro trimestre (76,67\% cartão e $68,15 \%$ prontuário). Quanto ao número de consultas realizadas por profissionais, a maior parte foi realizada por enfermeiras. Identifica-se que parte das mulheres não teve consulta com o médico durante o período de realização do pré-natal (48,33\% cartão e 48,28\% prontuário).

No que concerne às atividades prestadas na assistência pré-natal, os procedimentos de aferição de PA, dados antropométricos e vacinas foram os mais registrados, com preenchimento do cartão: 93,33\%, 78,33\% e 81,67\%, respectivamente e no prontuário: $86,96 \%, 76,81 \%$ e 73,91\%, respectivamente. Opondo-se ao mencionado, os dados sobre a inspeção das mamas (cartão 35\% e prontuário 40,58\%), realização de exame ginecológico (cartão $13,33 \%$ e prontuário $27,54 \%$ ) e participação de consultas odontológicas (cartão 18,33\% e prontuário 14,49\%), indicaram pouca realização.

Sobre os exames, conforme Tabela 1, verificou-se que, nas primeiras solicitações, os cartões possuíam percentual de registros maior do que os prontuários. Referente ao tipo, notam-se menores percentuais de registro para beta-HCG (cartão 66,67\%e prontuário 2,90\%), sorologias de toxoplasmose (cartão $56,67 \%$ e prontuário 52,17\%) e hepatite B (cartão $65 \%$ e prontuário 53,62\%). Encontraram-se poucos registros, não expressos em tabelas, referentes à realização do teste de tolerância oral à glicose-TTOG $(1,67 \%)$, parasitológicos de fezes $(1,67 \%)$, ureia/creatinina $(1,67 \%)$ e sorologia para rubéola $(3,33 \%)$.

Na Tabela 2, visualizam-se as repetições dos exames. Percebeu-se que o percentual de realização e consequentemente de registros são inferiores quando comparados aos de primeira solicitação (Tabela 1).

Os exames com maior frequência foram ABO/Rh e Anti-HIV com percentuais acima de $80 \%$. Nos outros tipos têm-se valores abaixo de $30 \%$, evidenciando-se as sorologias para toxoplasmose inferior a 10\% (Tabela 2).

Em relação à assistência domiciliar puerperal, registrados nos prontuários, verificou-se que foram realizadas $27,54 \%, 17,39 \%$ não foram realizadas e a falta de anotações alcançou $55,07 \%$. Referente aos profissionais que realizaram as visitas, $27,54 \%$ foram registradas por enfermeiras e o não preenchimento desse atendimento alcançou $72,46 \%$.

\section{- DISCUSSÃO}

Ao avaliar as variáveis de composição sociodemográfica, identificou-se o baixo percentual de preenchimento. Isto implica na subutilização destas informações, que poderiam contribuir para tomadas de decisões junto às usuárias durante o acompanhamento. A ausência dessas informações, no conjunto ou isolada, possui relação com a sua baixa utilização no cuidado pré-natal. Dessa forma, tal lacuna reflete em ineficiência na qualidade da assistência prestada, fato que pode levar ao aumento de morbimortalidade materno-fetal ${ }^{(11)}$.

Quanto à faixa etária, salienta-se a importância de conhecer a idade das usuárias gestantes, devido às complicações que podem surgir antecipando eventos em determinados períodos etários, possibilitando adequação da assistência conforme protocolos de condutas ${ }^{(12)}$. 
Tabela 1 - Distribuição dos exames de primeira solicitação. Lagoa Seca, PB, Brasil, 2014

\begin{tabular}{|c|c|c|c|c|}
\hline \multirow[t]{2}{*}{ Exames } & \multicolumn{2}{|c|}{ Prontuário } & \multicolumn{2}{|c|}{ Cartão da Gestante } \\
\hline & $\mathbf{n}$ & $\%$ & $\mathbf{n}$ & $\%$ \\
\hline \multicolumn{5}{|l|}{ 1. $\mathrm{ABO} / \mathrm{Rh} 1^{\mathrm{o}} \mathrm{t}^{*}$} \\
\hline Realizado & 52 & 75,36 & 56 & 93,33 \\
\hline Não realizado & 9 & 13,04 & 4 & 6,67 \\
\hline Sem registro & 8 & 11,59 & 0 & 0 \\
\hline \multicolumn{5}{|l|}{ 2. Glicemia $1^{\circ} t$} \\
\hline Realizado & 51 & 73,91 & 54 & 90 \\
\hline Não realizado & 9 & 13,04 & 6 & 10 \\
\hline Sem registro & 9 & 13,04 & 0 & 0 \\
\hline \multicolumn{5}{|c|}{ 3. Hemoglobina/Hematócrito $1^{\circ} \mathrm{t}$} \\
\hline Realizado & 58 & 84,06 & 58 & 96,67 \\
\hline Não realizado & 3 & 4,35 & 2 & 3,33 \\
\hline Sem registro & 8 & 11,59 & 0 & 0 \\
\hline \multicolumn{5}{|l|}{ 4. $\quad$ Anti-HIV $1^{\circ} \mathrm{t}$} \\
\hline Realizado & 50 & 72,46 & 52 & 86,67 \\
\hline Não realizado & 11 & 15,94 & 8 & 13,33 \\
\hline Sem registro & 8 & 11,59 & 0 & 0 \\
\hline \multicolumn{5}{|c|}{ 5. Hepatite $\mathrm{B} / \mathrm{HBsAg} 1^{\circ} \mathrm{t}$} \\
\hline Realizado & 37 & 53,62 & 39 & 65 \\
\hline Não realizado & 23 & 33,33 & 21 & 35 \\
\hline Sem registro & 9 & 13,04 & 0 & 0 \\
\hline \multicolumn{5}{|c|}{ 6. $\quad$ Sífilis/VDRL** $1^{\circ} \mathrm{t}$} \\
\hline Realizado & 55 & 79,71 & 57 & 95 \\
\hline Não realizado & 6 & 8,7 & 3 & 5 \\
\hline Sem registro & 8 & 11,59 & 0 & 0 \\
\hline \multicolumn{5}{|c|}{ 7. Toxoplasmose $1^{\circ} \mathrm{t}$} \\
\hline Realizado & 36 & 52,17 & 34 & 56,67 \\
\hline Não realizado & 24 & 34,78 & 26 & 43,33 \\
\hline Sem registro & 9 & 13,04 & 0 & 0 \\
\hline \multicolumn{5}{|c|}{ 8. $\quad$ Sumário de Urina $1^{\circ} \mathrm{t}$} \\
\hline Realizado & 53 & 76,81 & 51 & 85 \\
\hline Não realizado & 7 & 10,14 & 9 & 15 \\
\hline Sem registro & 9 & 13,04 & 0 & 0 \\
\hline \multicolumn{5}{|l|}{ 9. $\quad$ Beta-HCG } \\
\hline Realizado & 2 & 2,9 & 20 & 66,67 \\
\hline Não realizado & 1 & 1,45 & 40 & 33,33 \\
\hline Sem registro & 66 & 95,65 & 0 & 0 \\
\hline \multicolumn{5}{|c|}{ 10. Ultrassonografia } \\
\hline Realizado & 34 & 49,28 & 37 & 61,67 \\
\hline Não realizado & 20 & 28,99 & 23 & 38,33 \\
\hline Sem registro & 15 & 21,74 & 0 & 0 \\
\hline
\end{tabular}

${ }^{*} 1^{\circ}$ t: primeiro trimestre. ${ }^{* *} \mathrm{VDRL}=$ Venereal Disease Research Laboratory 
Tabela 2 - Distribuição dos exames de repetição realizado. Lagoa Seca, PB, Brasil, 2014

\begin{tabular}{|c|c|c|c|c|}
\hline \multirow[t]{2}{*}{ Exames } & \multicolumn{2}{|c|}{ Prontuário* } & \multicolumn{2}{|c|}{ Cartão da gestante } \\
\hline & $\mathbf{n}$ & $\%$ & $\mathbf{n}$ & $\%$ \\
\hline \multicolumn{5}{|l|}{ 1. $\mathrm{ABO} / \mathrm{Rh}$} \\
\hline Realizou & 6 & 8,7 & 58 & 96,67 \\
\hline Não realizou & 24 & 34,78 & 2 & 3,33 \\
\hline Sem registros & 31 & 44,93 & 0 & 0 \\
\hline \multicolumn{5}{|l|}{ 2. $\quad$ Glicemia } \\
\hline Realizou & 12 & 17,39 & 17 & 28,33 \\
\hline Não realizou & 18 & 26,09 & 43 & 71,67 \\
\hline Sem registros & 31 & 44,93 & 0 & 0 \\
\hline \multicolumn{5}{|c|}{ 3. Hemoglobina/Hematócrito } \\
\hline Realizou & 12 & 17,39 & 18 & 30 \\
\hline Não realizou & 18 & 26,09 & 42 & 70 \\
\hline Sem registros & 31 & 44,93 & 0 & 0 \\
\hline \multicolumn{5}{|l|}{ 4. $\quad$ Anti-HIV } \\
\hline Realizou & 8 & 11,59 & 51 & 85 \\
\hline Não realizou & 22 & 31,88 & 9 & 15 \\
\hline Sem registros & 31 & 44,93 & 0 & 0 \\
\hline \multicolumn{5}{|c|}{ 5. Hepatite B/HBsAg } \\
\hline Realizou & 7 & 10,14 & 7 & 11,67 \\
\hline Não realizou & 22 & 31,88 & 53 & 88,33 \\
\hline Sem registros & 32 & 46,38 & 0 & 0 \\
\hline \multicolumn{5}{|c|}{ 6. $\quad$ Sífilis/VDRL** } \\
\hline Realizou & 9 & 13,04 & 12 & 20 \\
\hline Não realizou & 21 & 30,43 & 48 & 80 \\
\hline Sem registros & 31 & 44,93 & 0 & 0 \\
\hline \multicolumn{5}{|c|}{ 7. $\quad$ Toxoplasmose } \\
\hline Realizou & 6 & 8,7 & 5 & 8,33 \\
\hline Não realizou & 23 & 33,33 & 55 & 91,67 \\
\hline Sem registros & 32 & 46,38 & 0 & 0 \\
\hline \multicolumn{5}{|c|}{ 8. $\quad$ Sumário de urina } \\
\hline Realizou & 12 & 17,39 & 12 & 20 \\
\hline Não realizou & 18 & 26,09 & 48 & 80 \\
\hline Sem registros & 31 & 44,93 & 0 & 0 \\
\hline
\end{tabular}

${ }^{*} \mathrm{O}$ complemento do $\mathrm{N}(69)$ equivale às participantes que ainda não haviam concluindo o terceiro trimestre de gestação, logo ainda poderiam vir a ser registrados após período de coleta da pesquisa, que totalizavam $8(11,59 \%)$. ${ }^{* *} \mathrm{VDRL}=\mathrm{Venereal}$ Disease Research Laboratory

Na pesquisa a maior frequência foi de mulheres casadas convivendo com companheiros e filhos, convergindo tal achado com pesquisas similares que referem a presença do companheiro como fator que contribui para a adesão aos serviços de saúde, relacionando aspectos psicológicos relativos à segurança e à estabilidade com adequação do pré-natal ${ }^{(13)}$.

No que diz respeito à escolaridade, predominou o ensino médio, nível de instrução considerado bom para contribuir com o desenvolvimento do pré-natal, devido a uma maior facilidade de compreensão das informações fornecidas ${ }^{(14)}$.

Para a variável raça, obteve-se neste estudo um insucesso nos dados preenchidos nos prontuários, alcançando $89,86 \%$ a ausência dessa informação. Uma pesquisa mostrou, ao avaliar o preenchimento 
da variável raça/cor em oito sistemas/módulos nacionais de informação em saúde, que existe um declínio abrupto no preenchimento desse campo, o que pode inviabilizar cálculos de indicadores relacionados ao enfoque étnico-racial, bem como identificar as diferenças e as necessidades reais, dificultando melhor acerto às políticas de promoção da equidade ${ }^{(15)}$.

De acordo com a ocupação informada, deparamo-nos com 50,72\% na agricultura, associando tal achado à residência na zona rural, seguidas das atividades interligadas às funções domésticas. É notória no mercado de trabalho brasileiro a distinção entre tarefas para mulheres e homens, compondo um cenário no qual as mulheres que trabalham fora do lar realizam ocupações tipicamente femininas, caracterizadas pela baixa remuneração e o desprestígio social refletindo sua posição na sociedade ${ }^{(16)}$.

Referente ao perfil obstétrico, a maioria das participantes teve uma a duas gestações. Estudo apontou maior prevalência de mulheres primigesta e secundigesta ${ }^{(17)}$. Para o número de filhos, houve predomínio de um filho, fato compreendido pela queda na taxa de fecundidade total no Brasil a qual a razão de fecundidade total (TFT) vem decrescendo. No ano 2000 alcançou 2,39 e em 2015 caiu para $1,72^{(18)}$.

Sobre o tipo de parto verificou-se que, tanto no anterior como no das gestações atuais, a maioria foi vaginal/normal. As mulheres com opção de realização do parto em setor público possuem proporção elevada do tipo de parto normal comparada a usuárias com assistência no setor privado, onde predomina o parto cesáreo ${ }^{(19)}$.

No que diz respeito à assistência pré-natal, que contribui com a avaliação da acessibilidade aos serviços de saúde, adotaram-se os critérios de qualidade norteados pelo Ministério da Saúde no programa Rede Cegonha ${ }^{(20)}$.

Para o número de consultas, o presente estudo deparou-se com mais de $70 \%$ das gestantes realizando seis ou mais consultas. Tal achado indica a possibilidade da gestante receber cuidados preventivos e de promoção da saúde durante as consultas ${ }^{(21)}$.

Face ao número de consultas pré-natal por profissional, observou-se a predominância de realização pelas enfermeiras. Contudo, o Ministério da Saúde propõe que, para uma boa assistência às gestantes, deve-se realizar as consultas de pré-natal intercaladas por enfermeiro(a) e médico(a)(10). Uma consulta de enfermagem não substitui uma consulta médica e vice-versa, portanto, é de suma importância o acompanhamento pelos dois profissionais.

Em relação ao início do pré-natal, observou-se o predomínio do início precoce, havendo, contudo, percentual expressivo de início tardio. Quanto ao início da assistência, a captação precoce das mulheres é de suma importância, pois propicia uma estimativa da idade gestacional mais fidedigna, com melhor acompanhamento do crescimento fetal e permite acesso a diagnósticos e terapias para diversas patologias com repercussões graves para a saúde da mulher e do bebê, como anemia, infecção pela sífilis e pelo HIV, hipertensão arterial crônica e diabetes ${ }^{(22)}$.

No tocante às atividades realizadas durante os atendimentos, as ações atribuídas aos profissionais técnicos de enfermagem (verificação da pressão arterial, vacinação, dados antropométricos) possuíam mais registros do que as realizadas por profissionais de nível superior (inspeção das mamas, exame ginecológico, consulta odontológica). Um estudo justificou achados semelhantes com o fato de profissionais de nível técnico terem uma formação para o agir mais tecnicista, realizando assistência numa prática mais reduzida a realizar tarefas ${ }^{(23)}$.

Foram perceptíveis os registros negligenciados durante todo o período de assistência do pré-natal, gerando dúvida se os campos deixados em branco sugerem a ausência de sua realização ou a falta de evolução pelo profissional. Pesquisa revelou que o exame ginecológico e a inspeção de mamas foram os procedimentos mais negligenciados, o que indica que essas práticas apresentam dificuldades em serem incorporadas à rotina do pré-natal, seja pelo tempo de consulta que demanda para a sua realização ou pela habilidade que é exigida do profissional ${ }^{(24)}$.

Sobre os exames, encontraram-se, neste estudo, lacunas referentes à realização e registros dos profissionais. Houve maior realização de hemograma, testagem para sífilis, fator Rh e menor nas sorologias para hepatite B e toxoplasmose. Tais achados podem ter relação com a organização do fluxo 
interno dos dois serviços estudados, que refletem em demora de marcação, entrega, dentre outros fatores inerentes à realidade estudada.

Os achados de realização das sorologias, essenciais para o sucesso da triagem durante o pré-natal, compõem os desafios a serem enfrentados nos serviços estudados. A não repetição dos exames aumenta o risco do nascimento de crianças infectadas, porque se perde a oportunidade de identificar a infecção aguda na gestante ${ }^{(24)}$.

Nesta direção, o planejamento assistencial municipal é essencial para o alcance dos exames do pré-natal voltados para as gestantes. Autores explicam que a disposição de laboratório municipal e agendamento organizado perante fluxograma, com comunicação entre os níveis de atenção, possibilitam a realização dos exames com rapidez ${ }^{(4)}$.

Quanto à visita domiciliar, este estudo identificou uma barreira que precisa ser revisada na assistência, expressa em percentual baixo dos profissionais das USF que realizaram consulta domiciliar puerperal. Um estudo também encontrou poucas visitas e as que foram realizadas perceberam que eram mulheres de alto risco(25).

Diante da análise dos cartões das gestantes e dos prontuários, percebe-se a existência de lacunas na assistência pré-natal nas unidades estudadas, evidenciada pela falta de registros em todas as variáveis coletadas nesta pesquisa, em ambas as fontes de coleta. Tais achados refletem na qualidade da assistência prestada nos serviços de saúde, uma vez que as anotações nesses documentos mostram os procedimentos realizados na consulta, porém é comum o sub-registro ${ }^{(23)}$.

\section{CONCLUSÃO}

Relativo à avaliação da assistência pré-natal, este estudo se deparou com lacunas nos serviços que evidenciam a deficiência da acessibilidade na dimensão organizacional, encontrando-se: deficiência nos registros de prontuários e cartões das gestantes; gestantes que não tiveram consulta de pré-natal com profissionais médicos(as); baixos percentuais dos procedimentos de inspeção de mamas, exame ginecológico e consulta odontológica, bem como baixa realização dos exames preconizados no programa Rede cegonha, vinculado ao município.

O estudo apresentou como limitação a ausência das informações nas fontes de coleta, principalmente as variáveis referentes às condições sociodemográficas e obstétricas. Dessa forma, não foi possível traçar um perfil mais representativo das atividades desenvolvidas pelos profissionais e realização de análises que verificassem as implicações dessas com as de assistência à mulher. Indica-se a realização de estudos que adotem procedimentos metodológicos que busquem as informações com as usuárias, profissionais e gestores, possibilitando o cruzamento das informações e compressão das diferentes faces dos serviços relacionadas à acessibilidade.

Pretende-se que esta pesquisa sirva de subsídio para gestoras(es), profissionais e usuárias(os) compreenderem o funcionamento das ações atuais, reconhecerem as barreiras de acessibilidade existentes nas USF e poderem criar estratégias para melhorar a assistência prestada durante o ciclo gravídico-puerperal.

\section{REFERÊNCIAS}

1. Dias MAB, Domingues RMSM, SchilithzAOC, Nakamura-Pereira M, Diniz CSG, Brum IR, et al. Incidência do near miss materno no parto e pós-parto hospitalar: dados da pesquisa Nascer no Brasil. Cad. Saúde Pública [Internet]2014; 30 (Suppl 1) [acesso em 25 mar 2015]. Disponível: http://dx.doi.org/10.1590/0102-311X00154213

2. Kassara SB, Melo AMC, Coutinho SB, Lima MC, Lira PIC. Determinants of neonatal death with emphasis on health care during pregnancy, childbirth and reproductive history. J. Pediatr. [Internet] 2013; 89(3) [acesso em 22 fev 2015]. Disponível: dx.doi.org/ 10.1016/j.jped.2012.11.005

3. de Castro ME, Moura MAV, da Silva LMS. Qualidade da assistência pré-natal: uma perspectiva das puérperas 
egressas. Rev. Rene. [Internet] 2010; 11(n.esp) [acesso em 11 mar 2015]. Disponível: http://www.revistarene.ufc. br/edicaoespeci al/a08v11 esp_n4.pdf

4. Duarte SJH, Mamede MV. Ações do pré-natal realizadas pela equipe de enfermagem na atenção primária à saúde, Cuiabá. Cienc. enferm. [Internet] 2013; 19(1) [acesso em 25 fev 2015]. Disponível: http://dx.doi.org/10.4067/ S0717-95532013000100011

5. Alves MLP. Adequação da atenção à Saúde da Mulher e da Criança no município do Paudalho segundo olhar da Rede Cegonha. Plano de Intervenção de Especialização em Gestão de Sistemas e Serviços em Saúde. Centro de Pesquisas Aggeu Magalhães. 2011. [acesso em 25 fev 2015]. Disponível: http://www.cpqam.fiocruz.br/ bibpdf/2012alves-mlp.pdf

6. Arakawa T, Arcêncio RA, Scatolin BE, Scatena LM, Ruffino-Netto A, Villa TCS. Acessibilidade ao tratamento de tuberculose: avaliação de desempenho de serviços de saúde. Rev. Latino-Am. Enfermagem [Internet] 2011; 19(4) [acesso em 22 fev 2015]. Disponível em: http://www.scielo.br/pdf/rlae/v19n4/pt_19.pdf

7. Fekete MC. Estudo da acessibilidade na avaliação dos serviços de saúde. In: Santana JP, organizadores. Desenvolvimento Gerencial de Unidades Básicas do Sistema Único de Saúde (SUS). Brasília: OPS; 1997. p. 84-177.

8. Barbatesfano OS, Girianelli VR, Muniz CV. O Acesso à assistência ao parto para gestantes e adolescentes nas maternidades rede SUS. Rev. Gaúcha Enferm. [Internet] 2010; 31(4) [acesso em 22 fev 2015]. Disponível: http:// dx.doi.org/10.1590/S1983-14472010000400014

9. Ministério da Saúde (BR). Cadernos de atenção básica. Atenção ao pré-natal de baixo risco. Brasília: Conselho Nacional de Saúde; Ministério da Saúde; 2012. 314-315 p.

10. Ministério da saúde (BR). Cartão da Gestante. Brasília: Conselho Nacional de Saúde; Ministério da Saúde; 2010.

11. Handell IBS, Cruz MM, Santos MA. Evaluation of antenatal care in selected Family Health Centres in a Midwest Brazilian municipality, 2008-2009. Epidemiol. Serv. Saúde [Internet] 2014; 23(1) [acesso em 25 mar 2015$].$ Disponível: http://dx.doi.org/10.5123/S1679-49742014000100010

12. Reticena KO, Mendonça FF. Perfil alimentar de gestantes atendidas em um hospital da Região Noroeste do Paraná. UNOPAR Cient. Ciênc. Biol. Saúde. [Internet] 2012; 14(2) [acesso em 15 mar 2015]. Disponível: http:// pgsskroton.com.br/seer//index.php/biologicas/article/view/1004/965

13. Barreto CN, Ressel LB, dos Santos CC, Wilhelm LA, da Silva SC, Alves CN. Atenção pré-natal na voz das gestantes. Rev enferm UFPE online. [Internet] 2013; 7(5) [acesso em 20 mar 2015]. Disponível em: http://www. revista.ufpe.br/revistaenfermagem/index.php/revista/article/download/4355/6373

14. Gaioso EMS, Santos FCS, Ferreira AGN, dos Santos LH, Santos Neto M, Santos FS. Gestantes atendidas em hospital de referência do SUS: quem são e quais os motivos?. J Manag Prim Health Care. [Internet] 2014; 5(1) [acesso em 16 mar 2015]. Disponível: http://www.jmphc.com/ojs/index.php/01/article/view/137/118

15. Braz RM, Oliveira PTR, Reis AT, Machado NMS. Avaliação da completude da variável raça/cor nos sistemas nacionais de informação em saúde para aferição da equidade étnico-racial em indicadores usados pelo Índice de Desempenho do Sistema Único de Saúde. Saúde debate [Internet] 2013; 37(99) [acesso em 20jul 2015]. Disponível: http://dx.doi.org/10.1590/S0103-11042013000400002

16. Neves AS, Santos MCB. Determinantes sociopolíticos e culturais e as repercussões sobre o pré-natal de alto risco: um olhar do Serviço Social sobre seu exercício profissional em um hospital universitário. Vértices [Internet] 2012; 14(1) [acesso em 25 mar 2015]. Disponível: http://www.essentiaeditora.iff.edu.br/index.php/vertices/article/ view/1809-2667.20120010/1342

17. Leite FMC, Barbosa TKO, Mota JS, Nascimento LCN, Amorim MHC, Primo CC. Perfil socioeconômico e obstétrico de puérperas assistidas em uma maternidade filantrópica. Cogitare enferm. [Internet] 2013; 18(2) [acesso em 20 fev 2015]. Disponível: http://dx.doi.org/10.5380/ce.v18i2.32584

18. Instituto Brasileiro de Geografia e Estatística (IBGE) [Internet]. Brasil em Síntese; [acesso em 18 jul 2015$].$ População-Taxa de fecundidade total-Brasil 2000 a 2015. Disponível: http://brasilemsintese.ibge.gov.br/ populacao/taxas-de-fecundidade-total.html 
19. Domingues PML, Nascimento ER, de Oliveira JF, Barral FE, Rodrigues QP, Santos CCC, et al. Discriminação racial no cuidado em saúde reprodutiva na percepção de mulheres. Texto contexto - enferm. [Internet] 2013; 22(2) [acesso em 20 jul 2015]. Disponível: http://dx.doi.org/10.1590/S0104-07072013000200003

20. Ministério da saúde (BR). Portaria n. 1.459, de 24 de junho de 2011: Institui, no âmbito do Sistema Único de Saúde - SUS - a Rede Cegonha. Diário Oficial da União, [Internet] 24 jun 2011 [acesso em 22 fev 2015]. Disponível: http://bvsms.saude.gov.br/bvs/saudelegis/gm/2011/prt1459_24_06_2011.html

21. Martinelli KG, Santos Neto ET, Gama SGN, Oliveira AE. Adequação do processo da assistência pré-natal segundo os critérios do Programa de Humanização do pré-natal e Nascimento e Rede Cegonha. Rev Bras Ginecol Obstet. [Internet] 2014; 36(2) [acesso em 25 mar 2015]. Disponível: http://dx.doi.org/10.1590/S0100-72032014000200003

22. Pereira NM, Guimarães BNS, Lanza FM. Avaliação da adequação da assistência pré-natal em uma unidade tradicional da atenção primária à saúde. R. Enferm. Cent. O. Min. [Internet] 2013; 3(3) [acesso em 20 jul 2015$].$ Disponível: http://www.seer.ufsj.edu.br/index.php/recom/article/view/405/527

23. Santos Neto ED, Oliveira AE, Zandonade E, Gama SGM, Leal MC. O que os cartões de pré-natal das gestantes revelam sobre a assistência nos serviços do SUS da Região Metropolitana da Grande Vitória, Espírito Santo, Brasil? Cad. Saúde Pública. [Internet] 2012; 28(9) [acesso em 24 mar 2015]. Disponível: http://dx.doi.org/10.1590/ S0102-311X2012000900005

24. Carellos EVM, de Andrade GMQ, de Aguiar RALP. Avaliação da aplicação do protocolo de triagem prénatal para toxoplasmose em Belo Horizonte, Minas Gerais, Brasil: estudo transversal em puérperas de duas maternidades. Cad. Saúde Pública. [Internet] 2008; 24(2) [acesso em 25 mar 2015]. Disponível: http://dx.doi. org/10.1590/S0102-311X2008000200018

25. Hass CN, Teixeira LB, Beghetto MG. Adequabilidade da assistência pré-natal em uma estratégia de saúde da família em Porto Alegre-RS. Rev. Gaúcha Enferm. [Internet] 2013; 34(3) [acesso em 24 mar 2015]. Disponível: http://dx.doi.org/10.1590/S1983-14472013000300003 